\title{
Stroke Epidemiology and Stroke Care Services in India
}

\author{
Jeyaraj Durai Pandian, ${ }^{a}$ Paulin Sudhan ${ }^{\mathrm{b}}$

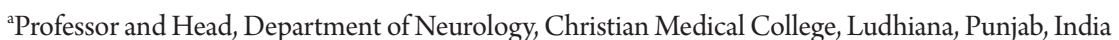 \\ ${ }^{b}$ Research Co-ordinator, Department of Neurology, Christian Medical College, Ludhiana, Punjab, India
}

Developing countries like India are facing a double burden of communicable and non-communicable diseases. Stroke is one of the leading causes of death and disability in India. The estimated adjusted prevalence rate of stroke range, 84-262/100,000 in rural and 334-424/ 100,000 in urban areas. The incidence rate is $119-145 / 100,000$ based on the recent population based studies. There is also a wide variation in case fatality rates with the highest being $42 \%$ in Kolkata. Stroke units are predominantly available in urban areas that too in private hospitals. Intravenous (IV) and intra-arterial thrombolysis (IA) are commonly used in India. In the on-going Indo USA National stroke registry the rate of IV thrombolysis is 11\%. Stroke rehabilitation is not well developed in India due to lack of personnel. Organised rehabilitation services are available in the country but they are mainly in private hospitals of the cities. Even though India is a leading generic drugs producer still many people can't afford the commonly used secondary prevention drugs. As a first step the Government of India has started the National Programme for Prevention and Control of Cancer, Diabetes, Cardiovascular Diseases \& Stroke (NPCDCS). The government is focusing on early diagnosis, management, infrastructure, public awareness and capacity building at different levels of health care for all the non-communicable diseases including stroke. An organised effort from both the government and the private sector is needed to tackle the stroke epidemic in India.

\author{
Correspondence: Jeyaraj Durai Pandian \\ Department of Neurology, Head of \\ Research, Betty Cowan Research and \\ Innovation Center, Christian Medical \\ College, Ludhiana, Punjab 141008, India \\ Tel: $+91-9915784750$ \\ Fax: +91-0161-2220850 \\ E-mail: jeyarajpandian@hotmail.com, \\ jeyarajpandian@yahoo.co.in
}

Received: August 1, 2013

Revised: September 4, 2013

Accepted: September 5, 2013

We sincerely thank Dr Benedict Joshua for his valuable contributions and $\mathrm{Mr}$ George Koshy final year medical student for his valuable help in the preparation of the Figure 1.

The authors have no financial conflicts of interest.

Keywords Stroke; India; Epidemiology; Incidence; Stroke unit; Rehabilitation

\section{Introduction}

Stroke is becoming an important cause of premature death and disability in low-income and middle-income countries like India, largely driven by demographic changes and enhanced by the increasing prevalence of the key modifiable risk factors. As a result developing countries are exposed to a double burden of both communicable and non-communicable diseases. The poor are increasingly affected by stroke, because of both the changing population exposures to risk factors and, most tragically, not being able to afford the high cost for stroke care. Majority of stroke survivors continue to live with disabilities, and the costs of on- going rehabilitation and long term-care are largely undertaken by family members, which impoverish their families. ${ }^{1,2}$

Studying the burden of stroke and the availability of health services will help the policy makers to tackle the rising burden of stroke. Recently there has been an increase in the epidemiology data on stroke from India. This review will address the changing burden of stroke and also the available stroke care services in India.

\section{Methods}

Papers were searched in the search engine PUBMED. The 
search terms used were 'Stroke in India,' 'Stroke epidemiology', 'Stroke burden', 'Stroke and India,, 'Stroke care in India', etc. Total number of papers listed was 1620 . Out of this around fifty papers were short listed and reviewed by the authors.

\section{Stroke Epidemiology during the pre-CT era}

Before the $\mathrm{CT}$ era, two population-based studies were undertaken in India. The first study was conducted in Vellore, Tamil Nadu, South India. This population-based study covering 258,576 people in and around Vellore was undertaken during the late 1960s and early 1970s. ${ }^{3}$ In the first phase (1968-1969), the number of hemiplegia cases was detected. In the second phase, this population was kept under surveillance for the next two years to record all cases of hemiplegia. ${ }^{3,4}$ This study revealed an incidence of 13/100,000 person-years and a point prevalence of $42 / 100,000$. The second study was conducted at Rohtak, Haryana, North India (1971-1974). Eighty-two cases of stroke were recorded yielding an annual incidence of 33/100,000 personyears. ${ }^{5}$ Unfortunately, no incidence study was reported from India over the next 2 decades.

\section{Stroke Epidemiology between the 1990s and 2010s Prevalence}

A stroke study conducted in Kolkata ${ }^{6}$ from 1998 to 1999 showed a crude prevalence rate of 147/100,000 and an annual incidence rate of $36 / 100,000$. When adjusted to the 1996 US population, the age-adjusted prevalence rate was $334 / 100,000$ and the age-adjusted annual incidence rate was 105/100,000. Compared to men, women had substantially higher age-adjusted prevalence rate $(564 / 100,000$ for women versus 196/100,000 for men) and incidence rate (204/100,000 for women versus $36 / 100,000$ for men). For all age groups except for people aged 50-69 years, women had a higher prevalence rate than did men. Among stroke patients who underwent neuroimaging study ( $59.5 \%$ of all strokes), $68 \%$ proved to be infarct and the remaining 32\% to be haemorrhage. Among risk factors for stroke assessed by case-control study, hypertension was the most important risk factor: odds ratio (OR) for hypertension was 5.04 (95\% confidence interval $[\mathrm{CI}]$ 4.16-5.92) for women and 21.87 (95\% CI 18.69-25.05) for men. Smoking in men had the OR 2.91 (95\% CI 1.57-4.25). However, the OR (95\% CI) for diabetes was not significant for both women (0.99 [0.28-2.26]) and men (1.61 [0.17-3.05]). Since the smoking prevalence in women was very low, the authors suggested that hypertension being less managed in women than in men might account for the higher incidence and prevalence in women. ${ }^{6}$

The prevalence of stroke in India shows a huge variation of $147-922 / 100,000$ across diverse community-based studies. ${ }^{4,7}$ In several studies which used age standardization with the US population as reference, the prevalence of stroke ranged from 244/ 100,000 to $424 / 100,000$.

According to the India stroke factsheet updated in 2012, the estimated age-adjusted prevalence rate for stroke ranges between $84 / 100,000$ and $262 / 100,000$ in rural and between $334 / 100,000$ and $424 / 100,000$ in urban areas. ${ }^{8}$

\section{Incidence}

World Health Organization (WHO) steps approach

In an effort to assist low-income and middle-income countries to establish surveillance systems for stroke, WHO recommended a stepwise approach (STEPS Stroke) through the use of standardized tools and methods for on-going core, expanded, and optional data collection. ${ }^{1}$ This system consists of three steps representing the possible outcomes of stroke patients in the hospital and the community. ${ }^{9}$

Step-1: The first step is gathering data from hospitalised patients such as demographic characteristics, whether it is the first ever or recurrent stroke, vital status at discharge, treatment during stay, risk factor assessment, classification of subtypes and follow up till discharge or death.

Step-2: The second level of survey involves identifying and gathering information about the non-hospitalised fatal stroke cases in the community after proper validation from death certificates, verbal autopsy or from direct autopsies.

Step-3: The third step represents non-fatal and non-hospitalized in the community and is the most complex level of stroke data collection.

In this section we have summarised data of four populationbased stroke epidemiology studies which were conducted according to the 'WHO-STEPS Stroke protocol' during the first decade of the 21st century in Mumbai, ${ }^{10}$ Trivandrum, ${ }^{11}$ Kolkata $^{12}$ and Bangalore ${ }^{13}$ areas (Figure 1). In the Mumbai study, which were conducted during a 2-year study period from January 2005 to December 2006, the crude annual incidence rate of first-ever stroke in people aged 25 years or more was $145 / 100,000$ person-years (age-standardized incidence rate, 152/100,000 person-years). ${ }^{10}$ In the Trivandrum study, which started its preparatory phase on January 2005 and completed its verification phase on August 2006, the crude annual incidence of any stroke was $117 / 100,000$ person-years (age-standardized incidence rate, $135 / 100,000$ person-years). ${ }^{11}$ In the Kolkata study using a doorto-door survey, the age-standardized incidence rate of first-everin-a-lifetime stroke was $145 / 100,000$ person-years. ${ }^{12}$

Age: It is assumed that the average age of patients with stroke in developing countries is usually 15 years younger than those in developed countries. In India, nearly one-fifth of patients with 


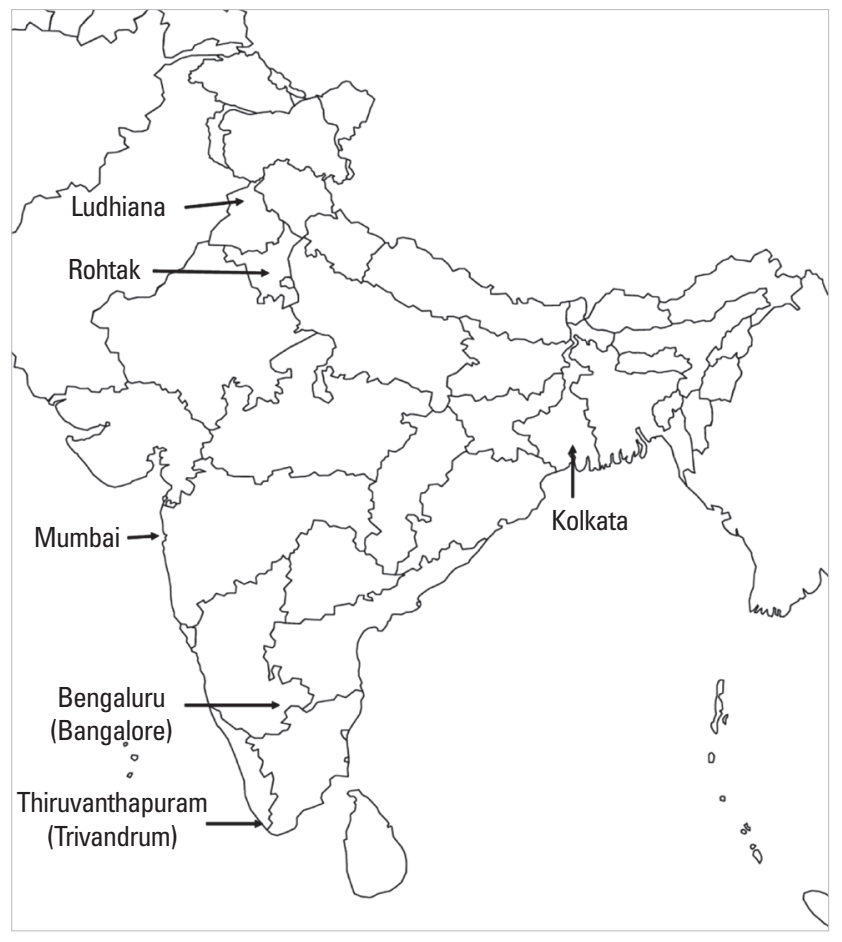

Figure 1. Map of India showing the different places where stroke incidence study has been done.

first-ever stroke admitted to hospitals has been estimated to be aged 40 years or less. But the Mumbai ${ }^{10}$ and Trivandrum ${ }^{11}$ registries showed that the mean age of patients with stroke was 66 and 67 years respectively. In contrast, in the Bangalore study the mean age was 54.5 years. ${ }^{13}$ In Trivandrum, stroke occurred at rate of 7.1 per 1000 per year in people aged $\geq 55$ years, and the rate escalated to 13.3 in people aged $\geq 75$ years (age-adjusted). ${ }^{11}$ The stroke in the young age group defined as 40 years or less comprised 3.8\%. In this study, the mean age of stroke onset did not differ between the urban and rural populations. ${ }^{11}$

Gender: In the Mumbai registry, men had a higher stroke incidence rate than did women (crude incidence rate, 149/100,000 person-years for men versus $141 / 100,000$ person-years for women; age-standardized incidence rate, 162/100,000 personyears for men versus $141 / 100,000$ person-years for women). ${ }^{10}$ Women were older ( 68.9 years) compared to men ( 63.4 years) ${ }^{10}$ In the Trivandrum registry, the crude incidence rate was higher in women than in men $(115 / 100,000$ person-years for men and $119 / 100,000$ person-years for women), but the age-standardized incidence rate was higher in men than in women (143/ 100,000 person-years for men and 128/100,000 person-years for women). ${ }^{11}$ The Bangalore study also showed a greater preponderance among men (67\%) with a male to female ratio of 2:1. The observed difference between age and gender and occurrence of stroke was statistically significant $(P<0.01){ }^{13}$

Stroke subtypes: Of patients with first-ever stroke captured in



Figure 2. Distribution of stroke subtypes in the various incidence studies.

the Mumbai registry, CT imaging was done in $89.2 \%$, and $80.2 \%$ were ischemic strokes and $17.7 \%$ hemorrhagic strokes (Figure 2). ${ }^{10}$ In the Trivandrum registry, $69.7 \%$ of patients underwent imaging. Of those, $83.6 \%$ were ischemic strokes, $11.6 \%$ intracerebral hemorrhages, and $4.8 \%$ subarachnoid haemorrhages, respectively. ${ }^{11}$ There were more strokes of undetermined type in patients enrolled from the rural communities because of a lack of neuroimaging information (31.2\%). ${ }^{11}$ In the Kolkata study, $32 \%$ of the patients had hemorrhagic stroke, which is the highest figure reported so far from India. ${ }^{12}$

Risk factors: It has been estimated that hypertension causes $54 \%$ of stroke in low-income and middle-income countries, followed by hypercholesterolemia (15\%) and tobacco smoking $(12 \%) \cdot{ }^{14}$ In the Mumbai registry, $82.8 \%$ of patients had hypertension. However, verifiable data for other risk factors were not available. ${ }^{10}$ In the Trivandrum registry, nearly $85 \%$ had hypertension, half had diabetes mellitus, $26 \%$ had dyslipidemia and $26.8 \%$ of men smoked tobacco. Compared to urban males, more rural males smoked tobacco ( $22.8 \%$ vs. $39.3 \%, P=0.013$ ). One risk factor was present in $38.4 \%$ patients, two in $42.0 \%$, and three or more in $14.4 \%$ patients. ${ }^{11}$

Urban vs Rural: Of the 541 validated first-ever strokes in Trivandrum, 431 occurred in the urban community and 110 occurred in the rural community. The annual stroke incidence rate was slightly higher in the rural population than in the urban population (crude incidence rate, 116/100,000 person-years for the urban population versus $119 / 100,000$ person-years for the rural population; age-standardized incidence rate, 135/100,000 person-years for the urban population versus $138 / 100,000$ person-years for the rural population) (Table 1). ${ }^{11}$ It also showed that the number of smokers (men) and presence of multiple 
risk factors (more than 3 ) were significantly more in rural population than in urban population. Also the distribution of conventional stroke risk factors was remarkably similar among the urban and rural communities. However number of stroke patients who had imaging was significantly low in rural population. ${ }^{11}$ Studies from India on cardiovascular risk factors have shown a 2 to 3 time's high prevalence of hypertension, hyperlipidemia, obesity, diabetes mellitus, and smoking (in men) in urban compared to rural communities. ${ }^{15,16}$

\section{Case fatality rate}

In the Mumbai study, the 28-day case fatality rate was $29.8 \%$ (Figure 3 ). ${ }^{10}$ The case fatality rate was $24.5 \%$ for urban and $37.1 \%$ for rural population (overall 27.2\%) in Trivandrum. ${ }^{11}$ Significantly more rural patients compared to urban patients died within 1 month, which probably reflects the disparity in the quality of acute stroke care between the rural and urban areas. ${ }^{11}$ The case fatality rate was $42 \%$ in the Kolkata study. ${ }^{12}$

Table 1. Data from Trivandrum stroke registry showing urban and rural distribution

\begin{tabular}{lccc}
\hline Factors & Urban & Rural & Pvalue \\
\hline Crude annual incidence rate & $116 / 100,000$ & $119 / 100,000$ & NS \\
Risk factors present & $94.9 \%$ & $94.1 \%$ & $\mathrm{NS}$ \\
Ischaemic stroke & $83.9 \%$ & $82.3 \%$ & $\mathrm{NS}$ \\
Hemorrhagic stroke & $16.1 \%$ & $17.7 \%$ & $\mathrm{NS}$ \\
Smoking (men) & $22.8 \%$ & $39.3 \%$ & 0.013 \\
3 or more risk factors & $12.7 \%$ & $20.8 \%$ & 0.034 \\
Imaging available & $71.9 \%$ & $56.4 \%$ & 0.003 \\
28 day fatality rate & $24.5 \%$ & $37.1 \%$ & 0.011 \\
28 day disability rate-Mild & $42.3 \%$ & $43.2 \%$ & NS \\
Moderate disability & $42.3 \%$ & $47.7 \%$ & $\mathrm{NS}$ \\
Severe disability & $15.4 \%$ & $9.1 \%$ & $\mathrm{NS}$ \\
\hline
\end{tabular}

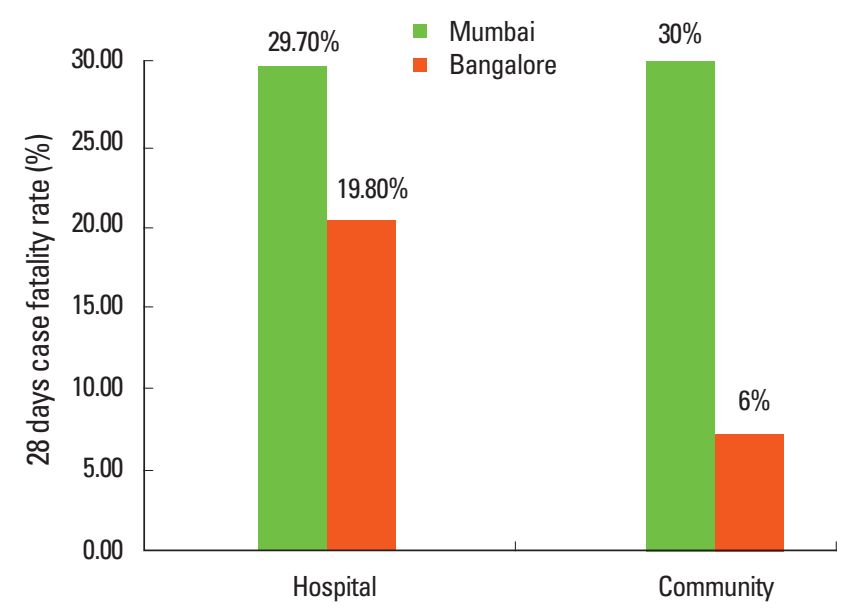

Figure 3. 28-day case fatality rates in hospital and community from the Mumbai and Bangalore registries.

\section{Disability}

In the Trivandrum study, the functional outcome was available in $86.8 \%$ among stroke survivors. ${ }^{11}$ The distributions of functional outcomes at 28 days among stroke survivors were mild disability (Rankin score 2 or less) in 42.4\%, moderate disability (Rankin score 3 or 4 ) in 43\%, and bedridden (Rankin score 5 ) in $14.6 \%$, respectively (Figure 4 ). Among stroke survivors, there was no significant difference in the functional outcome between men and women $(P=0.17)$, between urban and rural patients $(P=0.51)$, or across different age groups $(P=0.52) .{ }^{11}$

\section{Stroke in Ludhiana city}

In India the incidence studies on stroke have been done only in metropolitan cities so far. The Indian Council of Medical Research (ICMR) started a Task Force Project to develop population-based stroke registries across the country. As a pilot project, a population-based registry was conducted in Ludhiana city, Punjab. The crude annual incidence rate was found to be

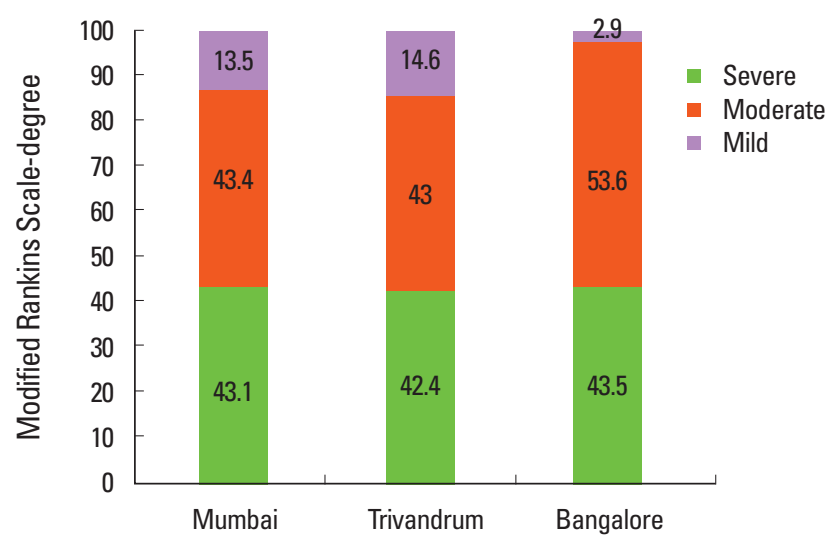

Figure 4. Distribution of post-stroke disability at 28 days from the onset of stroke among stroke survivors.



Figure 5. Stroke subtypes in the population-based Ludhiana stroke registry. 


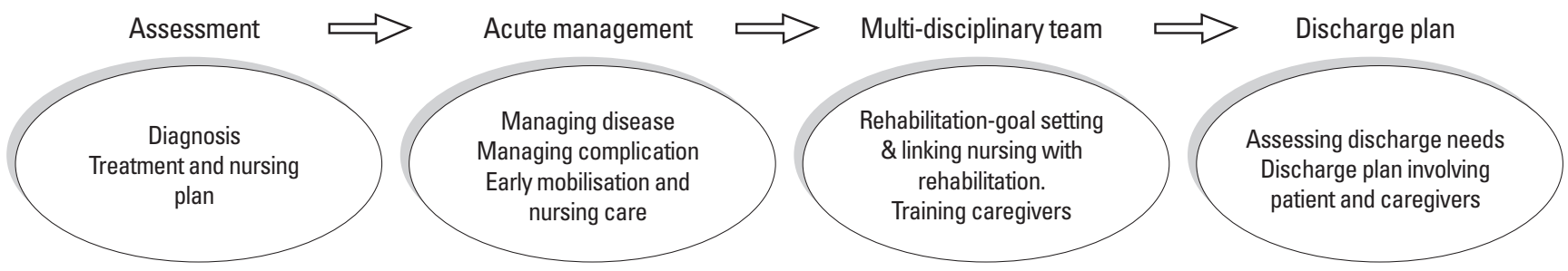

Figure 6. Components of stroke care unit.

140/100,000 person-years (95\% CI: 132.90 to 147.09 ) and the age-adjusted incidence rate was $181.67 / 100,000$ person-years (95\% CI: 172.44 to 190.89 ) (Unpublished data). Good outcome (defined as modified Rankin Scale $\leq 2$ ) was seen in 58\% of patients at 28 days. Case fatality rate at 28 days was $21.4 \%$. For stroke subtypes, ischemic stroke accounted for $73 \%$, intracerebral haemorrhage $21.7 \%$, and subarachnoid haemorrhage 4\% (Figure 5).

\section{Stroke care services in India}

Stroke units

Stroke unit is a multidisciplinary team comprising of medical, nursing, physiotherapy, occupational therapy, speech therapy, and social-work staff who coordinate their work through regular meetings (Figure 6). These meetings introduce the patients to the team and provide a forum for multidisciplinary assessment, identification of problems, and setting of short-term and long-term recovery goals. Stroke units typically include early active involvement of carers in the rehabilitation process and usually have a programme of on-going education and training. ${ }^{17}$ Two previous analyses that explored the potential population effect of different interventions for patients with stroke suggest that a basic model of stroke-unit care could provide the most effective population intervention. ${ }^{18}$

Stroke unit implementation remains a big challenge in India. At present in India there are approximately 35 stroke units and they are predominantly in private sector hospitals in the cities. Many of the private hospitals lack CT scan facility and they refer for incentives to other hospitals or centres. This results in crucial time being lost. On the other hand, public hospitals lack a dedicated team and place to manage stroke patients. Unavailability of CT scan is also an issue in smaller public hospitals as in private hospitals. According to the Mumbai registry study, only 306 of $456(67.2 \%)$ patients with first-ever stroke were managed at health-care facility ('in-hospital') and the remaining $150(32.8 \%)$ patients were cared at home or in nursing homes. ${ }^{10}$ This indicates that one out of every 3 patients with stroke are not accessing appropriate healthcare probably due to non-affordability, usage of alternative medicines, and difficulty in conveyance.

\section{Needs and Barriers}

We are facing many obstacles for implementing appropriate stroke care service in India. Stroke care units need a geographical base, a dedicated geographically defined hospital area with dedicated beds and nursing staff, though the need for expensive high-dependency facilities is less. ${ }^{18}$ An adequate number of skilled staff is an essential requirement of stroke-unit care and various skills will be needed to provide comprehensive care. In settings where skilled staff is few, options might include supplementary training for nursing staff, and training for family members and support workers. Constant medical supervision for early detection of post-stroke complication by nurses or trained stroke care physician is necessary for effective functioning of a stroke care unit which again demands a relatively bigger group of personnel to be involved. Last but not least is the requirement of adequate equipment and medication such as adequate bedding and seating, and equipment to assist with monitoring and fluid management. Some basic drugs are needed, particularly aspirin and antihypertensive drugs. Furthermore, generic drugs for lipid lowering and for diabetes management are important. These drugs which are already in the WHO list are yet to be made evenly available in India.

\section{Thrombolysis}

In India thrombolysis for stroke is being widely used across the country both in private and public sector hospitals. Compared to 2007, there has been an increase in the use of intravenous rtPA in the country (Figure 7). ${ }^{19,20}$ Even in the same center an increasing trend has been observed (Figure 8). ${ }^{21}$ The proportion of patients receiving rtPA is still low in our country. Among 967 patients enrolled in the on-going Indo-USA Collaborative National Stroke Registry, 134 patients came within 4.5 hours and 104 (11\%) patients received rtPA. Intraarterial and mechanical thrombolysis was given in 34 (3.5\%) patients. At present in India there are approximately 100 centres which are able to provide intravenous rtPA treatment and 55 centres capable of performing intraarterial or mechanical thrombolysis (Unpublished) 


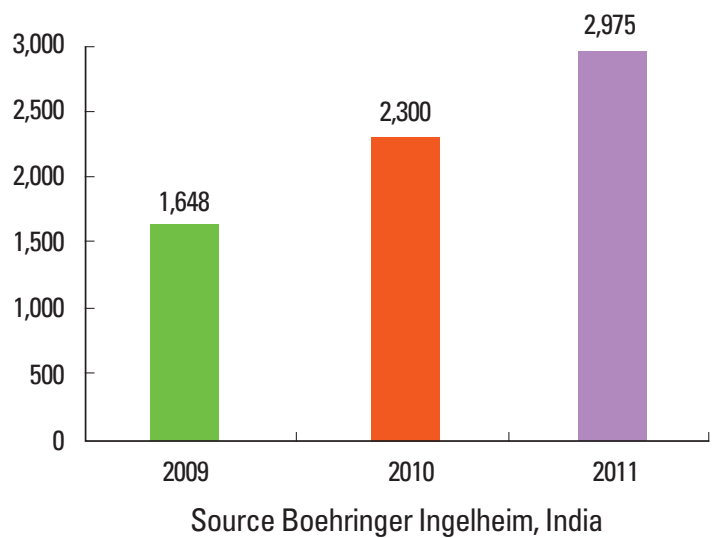

Figure 7. Nationwide data of numbers of patients treated with intravenous rtPA during 2009-2011.

\section{Rehabilitation}

In India the rehabilitation is mainly centred on physiotherapists. There are 32800 physiotherapists registered in Indian Association of physiotherapists till 2011, 3000 occupational therapists registered in Indian Association of Occupational therapists till 2011, and currently, 1700 speech therapists registered in Indian Association of speech therapists and 500 speech therapists registered in Rehabilitation Council of India. However, very few centers have an organized in-hospital and outpatient rehabilitation facilities in the country. Moreover use of complementary and alternative medicine treatments by the patients during the post stroke phase hampers the rehabilitation process. ${ }^{22}$

\section{Secondary prevention drugs}

Early initiation of treatments for secondary stroke prevention is associated with an $80 \%$ reduction in risk of early recurrent stroke. ${ }^{23} \mathrm{~A}$ standard secondary stroke prevention treatment will address multiple vascular risk factors and will usually consist of an antiplatelet agent, a lipid lowering drug mainly HMG-CoA reductase inhibitors (statins), and an antihypertensive agent. Monitoring of oral anticoagulant therapy is a major problem for cardioembolic stroke in India. The monitoring facilities are available mainly in the hospitals and the laboratories in the city. Newer oral aniticoagulants are used mainly in rich patients who could afford. Aspirin, clopidogrel and dipyridamole are the most commonly used antiplatelet drugs. Statins are also used with antihypertensive or antidiabetic drugs if indicated. India is the home of a thriving generic drug pharmaceutical industry. It is a thing to ponder on why poor people cannot afford modern therapies even where facilities are available. Even aspirin, although relatively cheap and readily available, is not routinely administered to patients with ischemic stroke or transient ischemic attack in India.

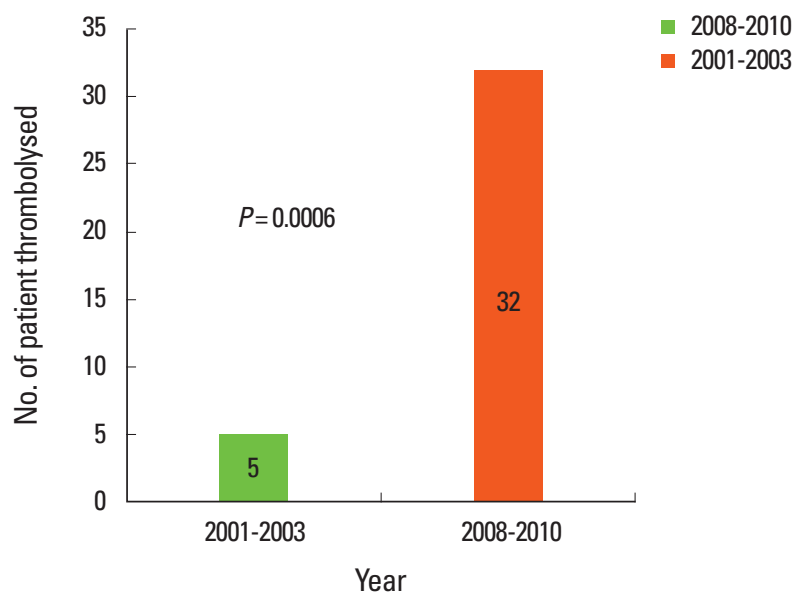

Figure 8. Secular trend of intravenous it PA treatment in Ludhiana city over two study periods.

\section{Conclusion}

India like other developing countries is in the midst of a stroke epidemic. There is a huge burden of stroke with significant regional variations. Stroke units, thrombolysis, and rehabilitation are predominantly available in urban areas, particularly in private sector hospitals. As a first step, the Government of India has started the National Programme for Prevention and Control of Cancer, Diabetes, Cardiovascular Diseases \& Stroke (NPCDCS). The government is focusing on early diagnosis, management, infrastructure, public awareness, and capacity building at different levels of health care for all the non-communicable diseases including stroke. An organised effort from both the government and the private sector is needed to tackle the rising stroke burden in India.

\section{References}

1. Bonita R, Beaglehole R. Stroke prevention in poor countries. Time for action. Stroke 2007;38:2871-2872.

2. Pandian JD, Srikanth V, Read SJ, Thrift AG. Poverty and stroke in India. A time to act. Stroke 2007;38:3063-3069.

3. Abraham J, Rao PS, Inbaraj SG, Shetty G, Jose CJ. An epidemiological study of hemiplegia due to stroke in South India. Stroke 1970;1:477-481.

4. Prasad K, Vibha D, Meenakshi. Cerebrovascular disease in South Asia - Part I: A burning problem. JRSM Cardiovascular Disease 2012;1:20.

5. Bansal BC, Prakash C, Jain AL, Brahmanandam KR. Cerebrovascular disease in young individuals below the age of 40 years. Neurol India 1973;21:11-18.

6. Banerjee TK, Mukherjee CS, Sarkhel A. Stroke in the urban 
population of Calcutta--and epidemiological study. Neuroepidemiology 2001;20:201-207.

7. Bharucha NE, Bharucha EP, Bharucha AE, Bhise AV, Schoenberg BS. Prevalence of stroke in the Parsi community of Bombay. Stroke 1988; 19:60-62.

8. http://www.sancd.org/Updated\%20Stroke\%20Fact\%20sheet \%202012.pdf: stroke fact sheet India. Accessed 21 July 2013.

9. Das SK. WHO Steps stroke surveillance system: Feasibility in India. Indian J Med Res 2009;130:359-360.

10. Dalal PM, Malik S, Bhattacharjee M, Trivedi ND, Vairale J, Bhat $\mathrm{P}$, et al. Population-based stroke survey in Mumbai, India: Incidence and 28-day case fatality. Neuroepidemiology 2008;31: 254-261.

11. Sridharan SE, Unnikrishnan JP, Sukumaran S, Sylaja PN, Nayak SD, Sarma PS, et al. Incidence, types, risk factors, and outcome of stroke in a developing country: The Trivandrum Stroke Registry. Stroke 2009;40:1212-1218.

12. Das SK, Banerjee TK, Biswas A, Roy T, Raut DK, Mukherjee $\mathrm{CS}$, et al. A prospective community-based study of stroke in Kolkata, India. Stroke 2007;38:906-910.

13. Nagaraja D, Gururaj G, Girish N, Panda S, Roy AK, Sarma GR, et al. Feasibility study of stroke surveillance: Data from Bangalore, India. Indian J Med Res 2009; 130:396-403.

14. Strong K, Mathers C, Bonita R. Preventing stroke: Saving lives around the world. Lancet Neurol 2007;6:182-187.

15. Singh RB, Sharma JP, Rastogi V, Raghuvanshi RS, Moshiri M, Verma SP, et al. Prevalence of coronary artery disease and coronary risk factors in rural and urban populations of North In- dia. Eur Heart J 1997; 18:1728-1735.

16. Goyal A, Yusuf S. The burden of cardiovascular disease in the Indian subcontinent. Indian J Med Res 2006;124:235-244.

17. Langhorne P, Pollock A, in conjunction with the Stroke Unit Trialists' Collaboration. What are the components of effective stroke unit care? Age Ageing 2002;31:365-371.

18. Langhorne P, Villiers LD, Pandian JD. Applicability of strokeunit care to low-income and middle-income countries. Lancet Neurol 2012;11:341-348.

19. Durai Pandian J, Padma V, Vijaya P, Sylaja PN, MurthyJM. Stroke and thrombolysis in developing countries. Int J Stroke 2007;2: 17-26.

20. Pandian JD, Sethi V, Dhillon R, Kaur R, Padala S, Chakravorty $\mathrm{R}$, et al. Is thrombolysis feasible in developing country? Cerebrovasc Dis 2006;20:134-136.

21. Pandian JD, Bhullar RS, Kaur P, Dhillon S, Toor G, Singh Y. Trends in the use of intravenous recombinant tissue plasminogen activator from northwest India. Int J of Stroke 2012;7:361365.

22. Pandian JD, Toor G, Arora R, Kaur P, Dheeraj KV, Bhullar RS, et al. Complementary and alternative medicine treatments among stroke patients in India. Top Stroke Rehabil 2012;19: 384-394.

23. Rothwell PM, Giles MF, Chandratheva A, Marquardt L, Geraghty $\mathrm{O}$, Redgrave JN, et al. Effect of urgent treatment of transient ischaemic attack and minor stroke on early recurrent stroke (EXPRESS study): A prospective population-based sequential comparison. Lancet 2007;370: 1432-1442. 